\title{
Renal perfusion evaluation by alternating current biosusceptometry of magnetic nanoparticles
}

\author{
Caio C. Quini ${ }^{\text {a,* }}$, Juliana F. Matos ${ }^{a}$, André G. Próspero ${ }^{a}$, Marcos Felipe F. Calabresi ${ }^{a}$, \\ Nicholas Zufelato ${ }^{\mathrm{b}}$, Andris F. Bakuzis ${ }^{\mathrm{b}}$, Oswaldo Baffa ${ }^{\mathrm{c}}$, José Ricardo A. Miranda ${ }^{\mathrm{a}}$ \\ a Departamento de Física e Biofísica, Instituto de Biociências, UNESP, Botucatu, SP, Brazil \\ ${ }^{\mathrm{b}}$ Instituto de Física, UFG, Goiânia, GO, Brazil \\ ${ }^{\mathrm{c}}$ Departamento de Física, FFCLRP, USP, Ribeirão Preto, SP, Brazil
}

\section{A R T I C L E I N F O}

Article history:

Received 27 June 2014

Received in revised form

29 September 2014

Available online 6 October 2014

\begin{abstract}
A B S T R A C T
Alternating current susceptometry, a simple and affordable technique, was employed to study the sensitivity of this approach to assess rat kidney perfusion by the injection of $200 \mu \mathrm{L}$ of magnetic nanoparticles with a concentration of $23 \mathrm{mg} / \mathrm{mL}$ in the femoral vein and the measurement of the signal above the kidney. The instrument was able to detect the signal and the transit time of the first and second pass were measured in five animals with average values of $13.6 \pm 4.3 \mathrm{~s}$ and $20.6 \pm 7.1 \mathrm{~s}$.
\end{abstract}

(c) 2014 Elsevier B.V. All rights reserved.

\section{Introduction}

Magnetic particles have a tremendous potential for medical diagnostics and therapy and many applications have been reported [1]. Because the human body is essentially transparent to the magnetic fields and the magnetic fields can interact with the human tissue in different ways one can envisage many application possibilities, such as hyperthermia, drug delivery, cell separation, magnetic resonance contrast changes, magnetic particle imaging and its transit through different organs, among many others. In this perspective, it can be expected that in the future, many studies that are currently performed with a radioactive tracers would be also feasible with magnetic tracers with the convenience of no radiation risk and all the associated benefits. Magnetic microparticles (diameter $\sim 100 \mu \mathrm{m}$ ) have been used to study gastrointestinal motility [2] and in the pharmaceutical sciences [3-5] in our group with a relatively simple instrumentation. Trillaud et al. [6] showed that renal perfusion in rabbits can be evaluated with firstpass study of superparamagnetic iron oxide particles (SPIO) using magnetic resonance fast imaging techniques. These authors pointed out the advantages of using nanoparticles instead of the commonly used gadolinium chelates that have a vascular phase that can be obscured by interstitial diffusion and glomerular filtration of the contrast agent. Differently, magnetic nanoparticles remain in the blood pool during the first pass because these

\footnotetext{
* Corresponding author.

E-mail address: caioquini@ibb.unesp.br (C.C. Quini).
}

particles are not eliminated by the kidney and show no interstitial diffusion [7,8].

Thus, the goal of this work is to show the potential of this instrumentation to study the transit of magnetic tracers in the rat kidney trough the signal time evolution obtained with an $A C$ biosusceptometer (ACB).

\section{Materials and methods}

\subsection{Biosusceptometric technique}

A single ACB sensor works as a double magnetic flux transformer with an air nucleus and has two coaxial pairs of coils separated by a baseline (b) of $15 \mathrm{~cm}$ [9]. Each pair is composed of an excitation coil (outer) with $N_{\mathrm{e}}$ coils and area $A_{\mathrm{e}}$ and a detection coil (inner) with $N_{\mathrm{d}}$ coils and area $A_{\mathrm{d}}$ arranged in a firstorder gradiometric configuration that provides the cancelation of the common mode signal leading to good signal-to-noise ratio. One pair works as the reference and the other as the detector probe. The excitation coil works with a frequency of $10 \mathrm{kHz}$ generated by a lock-in amplifier fed to a power amplifier that supplies a current of $88 \mathrm{~mA}$ which produces a magnetic field of $20 \mathrm{G}$ (rms) and induces equal magnetic flux in the detection coils. Hence, when a magnetic sample with volume $\delta v$ and magnetic susceptibility $\chi$ is near one extreme of the sensor, an imbalance in the voltage occurs, due to the changes in the flux between the detection coils. The following expression shows how the flux will change as a function of the parameters. It can be seen that a change $\delta V$ in the volume of the magnetic material will change the 
flux $\Phi$ and therefore the voltage detected.

$\Delta \Phi=\frac{N_{s} N_{e} \chi \mu_{0} \delta V\left(A_{s} A_{e}\right) I}{4 \pi}\left[\frac{1}{r^{6}}-\frac{1}{(r+b)^{6}}\right]$

The ACB sensor can locate and quantify the magnetic material through magnetic flux variation between the set of coils. The signal intensity detected by the sensors depends on the area of the detection coil, number of turns, magnetic flux change rate (i.e. applied field and frequency), the amount of magnetic material as well as the distance between the sensor and the magnetic sample. For this study, the ACB sensor was developed with these characteristics: excitation coils diameter $\varphi=3.5 \mathrm{~cm} ; 200$ turns of 26AWG wire and detection coils diameter $\varphi=2.9 \mathrm{~cm} ; 500$ turns of 32-AWG wire, to improve spatial resolution and sensitivity for laboratory animals. Sensitivity tests and a resolution profile were made using a small test tube containing $200 \mu \mathrm{L}$ of magnetic nanoparticles.

\subsection{Animals}

Five male Wistar rats weighing from 200 to $250 \mathrm{~g}$ were employed in this experiment. The animals were obtained from the institutional vivarium and were fed ad libitum before the experiment. All procedures were performed in accordance with the Guide of the Care and Use of Laboratory Animals (Brazilian College of Animal Experimentation) and were approved by the local Animal Ethics Committee under protocol number 409-CEUA.

\subsection{Nanoparticles}

The magnetic nanoparticles (MNp) used were synthesized by a coprecipitation procedure previously described [10] and consisted of manganese ferrite-based (MNF-citrate) nanoparticles surfacecoated with citric acid, which has already been tested for toxicity levels showing to be safe for animal injection under this concentration [11].

Fig. 1 shows the X-ray diffraction pattern of the citrate-coated manganese-ferrite nanoparticle. The data confirms the spinel structure and revealed a crystalline diameter of $16.7 \mathrm{~nm}$ after using Scherrer's relation.

The MNF-citrate nanoparticle size distribution was obtained using images from a transmission electron microscope (TEM) (Fig. 2) from JEOL model JEM-2100 operating at $200 \mathrm{kV}$ (resolution

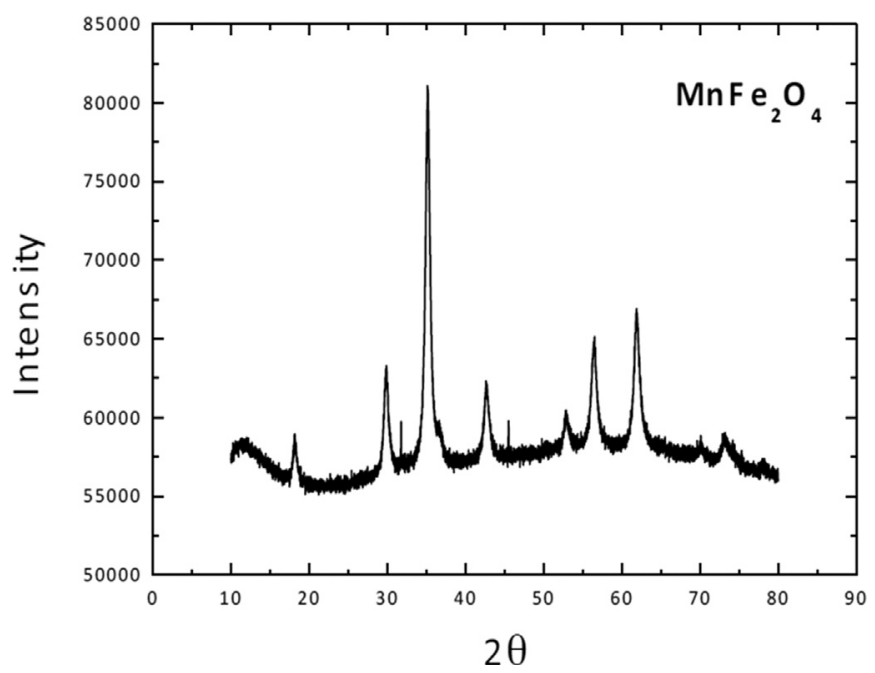

Fig. 1. X-ray diffraction pattern of the citrate-coated manganese-ferrite nanoparticles.

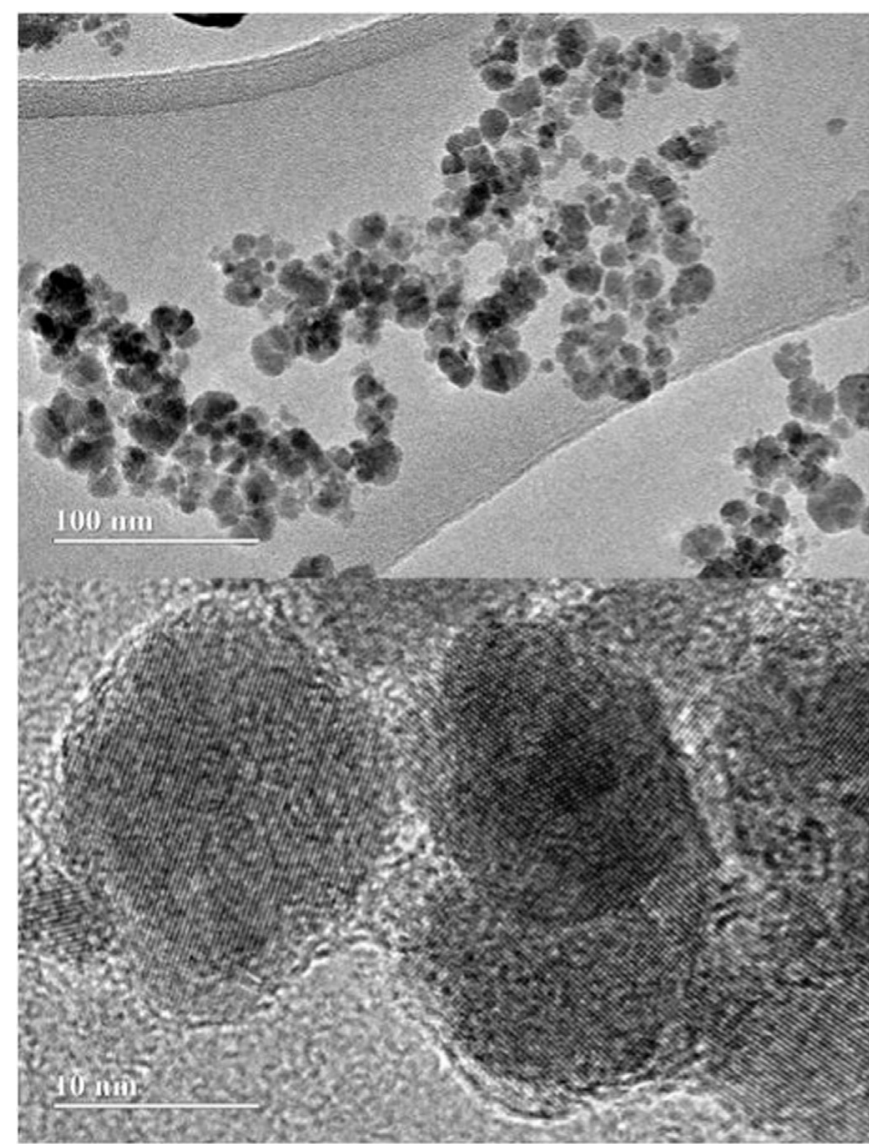

Fig. 2. TEM images for the magnetic nanoparticles. Scale: (A)100 and (B)10 nm.

$2.5 \AA$ ). We found a mean particle size of $13 \pm 4 \mathrm{~nm}$, shown in Fig. $3 \mathrm{~A}$.

The dynamic light scattering experiment was performed using a Zetasizer NanoS from Malvern. Fig. 3B below shows the hydrodynamic radius of the magnetic fluid sample from which we found a mean size of $273 \mathrm{~nm}$ with a PDI of 0.210 .

As the ACB system is based on acquisitions of magnetic flux variations and consequently, the $A C$ susceptibility, so the nanoparticles used as magnetic tracers needed to be highly susceptible to magnetic induction. The AC Susceptibility for Manganese Ferrite nanoparticles is considerably higher than for the iron oxide particles, which allowed using a considerable smaller magnetic particle concentration to acquire the same signal. Fig. 4 shows the magnetization curve of the manganese-ferrite based nanoparticles obtained using an ADE Vibrating Sample Magnetometer model EV9. The saturation magnetization of the nanoparticles was found to be $52.8 \mathrm{emu} / \mathrm{g}\left(264 \mathrm{emu} / \mathrm{cm}^{3}\right)$. The data indicates that the nanoparticles behave as a quasi-static superparamagnet, i.e. the nanostructure does not show any coercive field at DC conditions. The particle concentration was determined from the analysis of the magnetic fluid magnetization data. From this analysis we estimated that the NP stock solution had a concentration of $23 \mathrm{mg} / \mathrm{ml}$. The soft-ferrite based magnetic nanoparticle was chosen due to its good response at low magnetic field amplitude [12].

\subsection{Procedure}

Animals were anesthetized by intramuscular injection of ketamine (Ketamina Agener, $30 \mathrm{mg} / \mathrm{kg}$, União Química Farmacêutica Nacional, Embu-Guaçu, Brazil) and xylazine (Anasedan, $15 \mathrm{mg} / \mathrm{kg}$, 
A

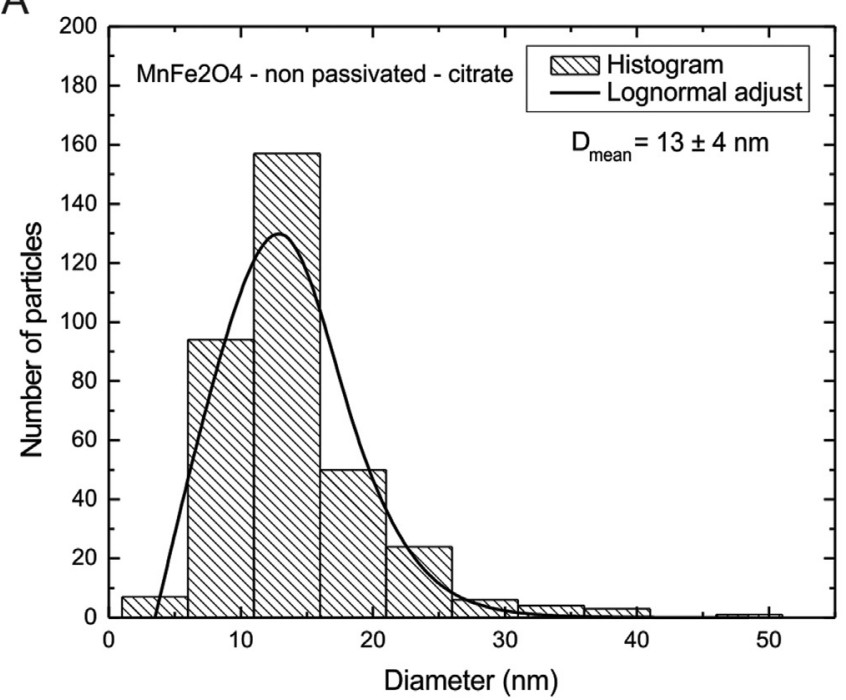

B

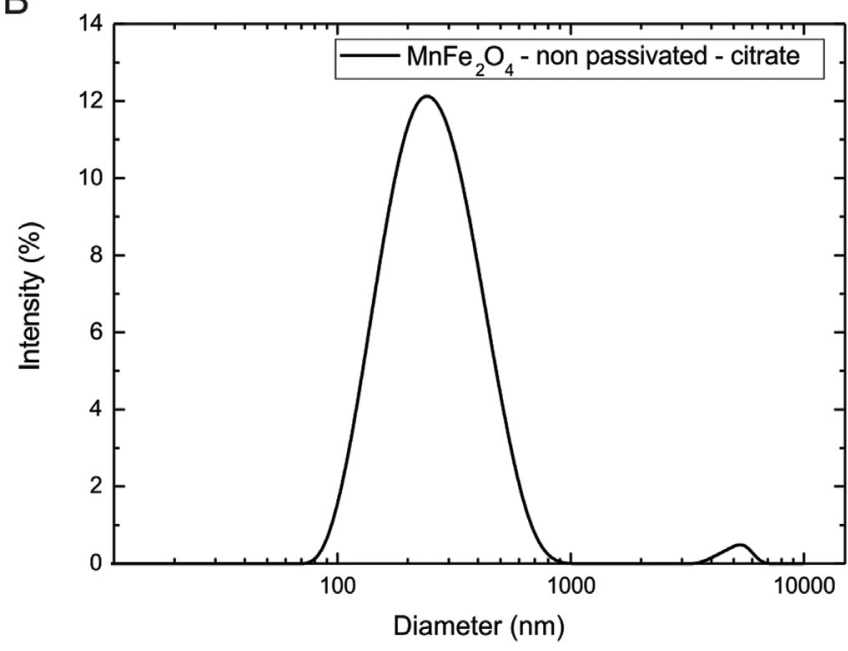

Fig. 3. Particles size distribution (A) and Hydrodynamic distribution (B) for the magnetic nanoparticles.

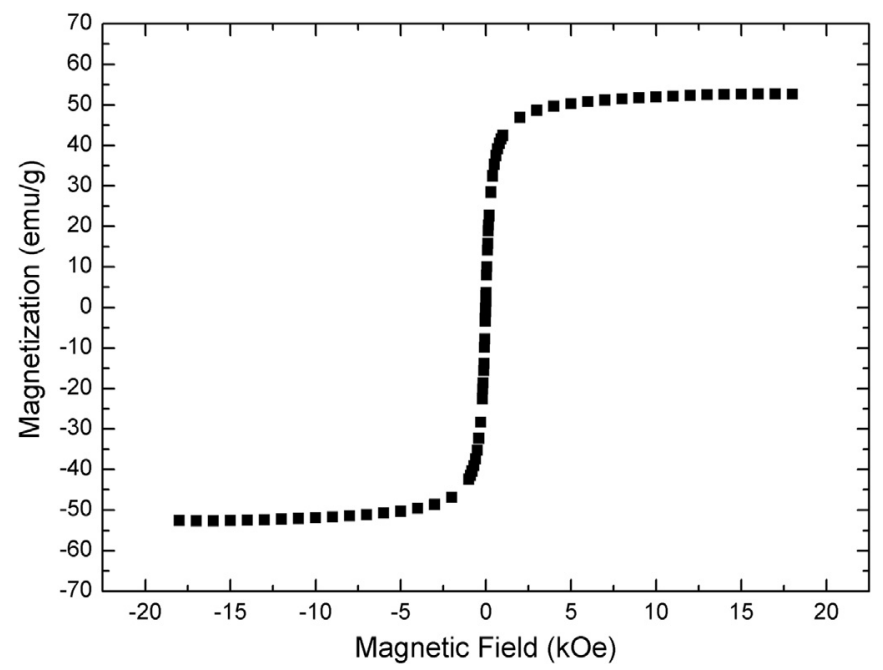

Fig. 4. Magnetization curve of the manganese-ferrite nanoparticles.

Vetbrands, Paulínia, Brazil). After anesthesia, the animal was positioned as shown in Fig. 5, with the sensor close to the lumbar back projection of the kidney.

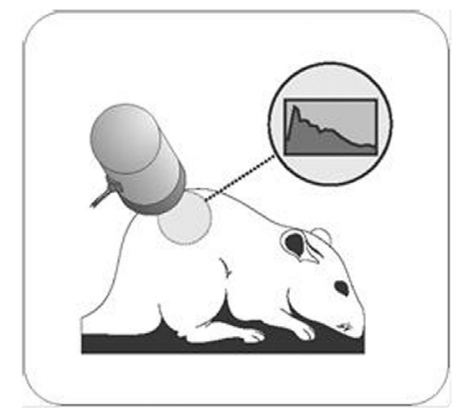

Fig. 5. Positioning the sensor to acquire the magnetic signal from the kidney.

A volume of $200 \mu \mathrm{L}$ of magnetic tracer at a concentration of $23 \mathrm{mg} / \mathrm{mL}$ was manually administered in approximately $5 \mathrm{~s}$ by infusion followed by $0.2 \mathrm{~mL}$ of pure saline solution into the right femoral vein. To ensure that the acquired signal does not suffer interference from nearby organs, a small incision was made in the lumbar posterior surface of each animal, allowing the externalization of the kidney. However, measurements made without this externalization also showed satisfactory performance.

\subsection{Data analysis}

All raw signals were processed in MatLab (Mathworks, Inc., USA) by digital filtering with a low pass second order Butterworth filter at $100 \mathrm{~Hz}$ and baseline correction. The transit time of the first and second pass was measured as the width from the signal onset and the first two peaks detected and are expressed as mean \pm standard deviation (SD).

\section{Results and discussion}

Fig. 6 shows the set up for the sensor sensitivity test (4A), for changes in distance to the magnetic material, and spatial resolution test (4B), when the test tube containing the same volume of MNPs is moved laterally at $1 \mathrm{~cm}$ of distance from the sensor.

Fig. 7 shows the sensitivity profile for the signal versus distance test.

A slow decay can be noticed for distances lower than $1 \mathrm{~cm}$ and for distances greater than $1 \mathrm{~cm}$ the decay follows approximately the simplified function described in the Equation.

Fig. 8 shows the spatial resolution when the test tube containing the same volume of MNPs is moved laterally at $1 \mathrm{~cm}$ of distance from the sensor. The expected shape of the curve is obtained [13] with a resolution of $1.85 \mathrm{~cm}$, measured as the width at the half maximum of the curve.

Fig. 9 shows one representative plot of the signal versus time obtained in this experiment. In all data, it is possible to observe the arrival of the MNPs at the kidney, the increase in signal intensity indicating the collection of MNPs in the organ, its clearance and the second passage. The signal does not return to its base line and the possible reason is the perfusion and collection of the magnetic nanoparticles in the kidney. The focus was to analyze the first

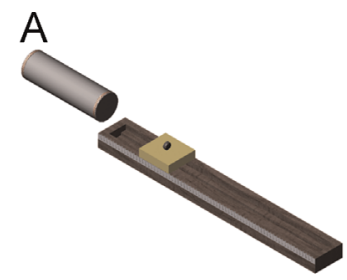

B

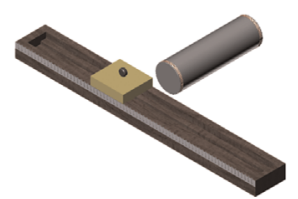

Fig. 6. Set up for the sensitivity test (A) and for the spatial resolution one (B). 


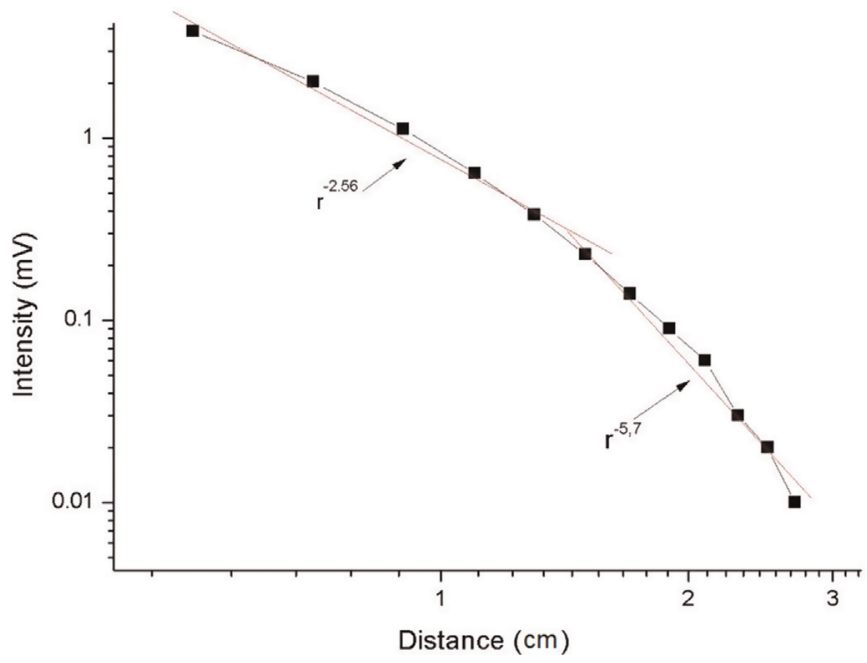

Fig. 7. Intensity of the signal detected by the biosusceptometer as a function of the distance for a $200 \mu \mathrm{L}$ of the MNPs used in the experiment.

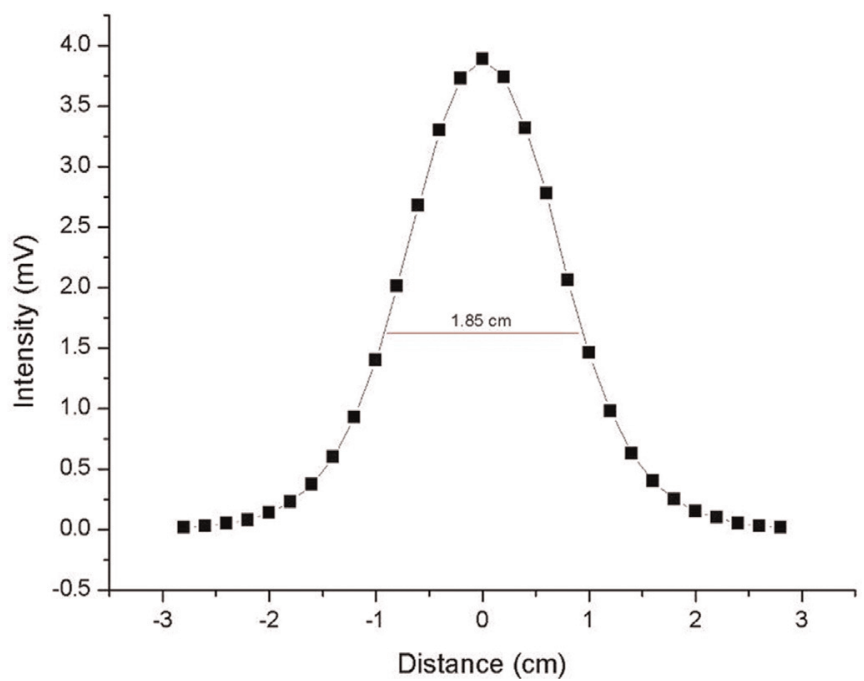

Fig. 8. Intensity of the signal detected by the biosusceptometer as a function of the distance for a $200 \mu \mathrm{l}$ of the MNPs used in the experiment.

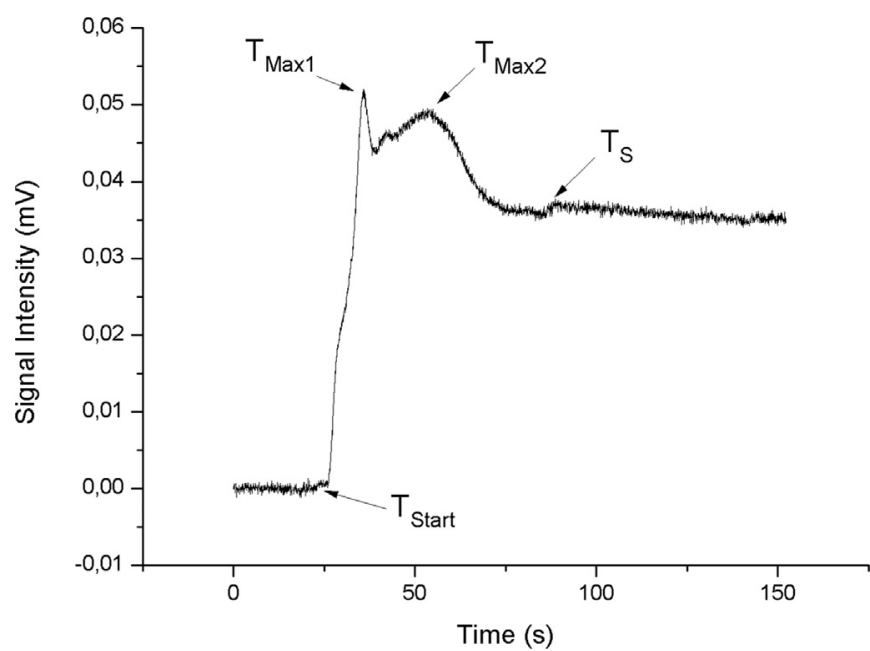

Fig. 9. Representative plot of the signal versus time obtained from five animals studied.
Table 1

Time intervals observed in the 5 animals after MNPs infusion.

\begin{tabular}{llllll}
\hline Animal & $T_{\max 1}(\mathrm{~s})$ & $T_{\max 2}(\mathrm{~s})$ & $T_{\text {int }}(\mathrm{s})$ & $\mathrm{I}_{\mathrm{S}}(\mathrm{mV})$ & $T_{\mathrm{s}}(\mathrm{s})$ \\
\hline 1 & 15 & 32 & 17 & 0.95 & 77 \\
2 & 13 & 35 & 22 & 0.9 & 80 \\
3 & 11 & 30 & 19 & 1.4 & 65 \\
4 & 11 & 41 & 30 & 1.45 & 75 \\
5 & 12 & 37 & 25 & 1.5 & 70 \\
Mean & 12.4 & 35 & 22.6 & 1.24 & 73.4 \\
SD & 1.7 & 4.3 & 5.1 & 0.29 & 5.9 \\
\hline
\end{tabular}

three minutes after MNPs infusion, however longer measurements indicate a tendency to return to baseline levels. The first pass is described as the interval between injection time $\left(T_{\text {Start }}\right)$ and the first peak $\left(T_{\max 1}\right)$. Second pass corresponds to the injection and second peak interval $\left(T_{\max 2}-T_{\text {Start }}\right)$. The time interval between passages $\left(T_{\text {Int }}\right)$ was also recorded, been the difference between second and first passage $\left(T_{\max 2}-T_{\max 1}\right)$. As the signal intensity does not return to the background value, the intensity in which the signal stabilizes $\left(I_{\mathrm{S}}\right)$ and the instant that occurs $\left(T_{\mathrm{S}}\right)$ was also recorded. The average time for the first pass was $13.6 \pm 4.3 \mathrm{~s}$, range $10 \mathrm{~s}$ to $21 \mathrm{~s}$ and for the second pass it was $20.6 \pm 7.1 \mathrm{~s}$, range 13$32 \mathrm{~s}$. Table 1 specifies all these data for each animal subjected to the test.

\section{Discussion}

The curve shown in Fig. 6 is very similar to those obtained by the fitting of the perfusion curves of the kidney in MRI studies [8]. The curve of the kidney exhibits a typical shape: an early peak corresponds to the first pass of the magnetic nanoparticles and second broader peak can be attributed to the recirculation. However, contrary to other contrast agents that can be transferred to the glomerular-tubular system, diminishing considerably this second peak, in our case it is still prominent. Trillaud et al. [6] used SPION as a contrast agent and measured the first peak in rabbits, that for MRI is negative due to the increase in the relaxivity of the tissues. The values they found are higher than those found in our study, but this could be explained by the difference of the experimental protocol, different animals, injections of MNPs and type of MNPs used are some possibilities. Regarding the use of citrate-coated nanoparticles, studies with rats and mice had not revealed any toxicity related problems under the doses used so far $[11,12]$. Another point is the resolution of the susceptometer, although the organ was exteriorized it is possible that signal from the vasculature that fed the kidney could also be captured that would increase the transit time measured. After developing methods to study these patterns in other organs, using different particles and also different coating materials, will be possible to combine an optimized $A C B$ sensor, specific magnetic particle and also specific targeting techniques, to study tissue targeting and drug delivery systems within a nanoscale accuracy.

\section{Conclusions}

The AC biosusceptometer has adequate sensitivity to detect the presence of the MNps in the kidney and dynamic studies of the kidney activity can be performed. 


\section{Acknowledgements}

Partial financial support: FAPESP Grant 2010/07639-9 and 2011/ 18696-6, FAPEG, CNPq and CAPES

\section{References}

[1] A. Ito, M. Shinkai, H. Honda, T. Kobayashi, Medical application of functionalized magnetic nanoparticles, J. Biosci. Bioeng. 100 (2005) 1-11.

[2] J.R. Miranda, O. Baffa, R.B. de Oliveira, N.M. Matsuda, An AC biosusceptometer to study gastric emptying, Med. Phys. 19 (1992) 445-448.

[3] L.A. Cora, P.R. Fonseca, M.F. Americo, R.B. Oliveira, O. Baffa, J.R. Miranda, Influence of compression forces on tablets disintegration by AC Biosusceptometry, Eur. J. Pharm. Piopharm.: Off. J. Arb. Pharm. Verfahr. 69 (2008) 372-379.

[4] L.A. Cora, F.G. Romeiro, M. Stelzer, M.F. Americo, R.B. Oliveira, O. Baffa, J. R. Miranda, AC biosusceptometry in the study of drug delivery, Adv. Drug Deliv. Rev. 57 (2005) 1223-1241.

[5] M.F. Americo, R.B. Oliveira, F.G. Romeiro, O. Baffa, L.A. Cora, J.R. Miranda, Scintigraphic validation of AC Biosusceptometry to study the gastric motor activity and the intragastric distribution of food in humans, Neurogastroenterol. Motil.: Off. J. Eur. Gastrointest. Motil. Soc. 19 (2007) 804-811.

[6] H. Trillaud, P. Degreze, Y. Mesplede, C. Douws, J. Palussiere, N. Grenier, Evaluation of experimentally induced renal hypoperfusion using iron oxide particles and fast magnetic resonance imaging, Acad. Radiol. 2 (1995) 293-299.

[7] O. Baffa, R.B. Oliveira, J.R. Miranda, L.E. Troncon, Analysis and development of AC biosusceptometer for orocaecal transit time measurements, Med. Biol. Eng. Comput. 33 (1995) 353-357.

[8] M. Notohamiprodjo, M.F. Reiser, S.P. Sourbron, Diffusion and perfusion of the kidney, Eur. J. Radiol. 76 (2010) 337-347.

[9] N.A. Daghastanli, F.J.H.N. Braga, R.B. Oliveira, O. Baffa, Oesophageal transit time evaluated by a biomagnetic technique, Physiol. Meas. 19 (1998) 413-420.

[10] L.C. Branquinho, M.S. Carriao, A.S. Costa, N. Zufelato, M.H. Sousa, R. Miotto, R. Ivkov, A.F. Bakuzis, Effect of magnetic dipolar interactions on nanoparticle heating efficiency: implications for cancer hyperthermia, Sci. Rep. 3 (2013) 2887.

[11] A.D. Nunes, L.S. Ramalho, A.P. Souza, E.P. Mendes, D.B. Colugnati, N. Zufelato M.H. Sousa, A.F. Bakuzis, C.H. Castro, Manganese ferrite-based nanoparticles induce ex vivo, but not in vivo, cardiovascular effects, Int. J. Nanomed. 9 (2014) 3299-3312.

[12] H.F. Rodrigues, F.M. Mello, L.C. Branquinho, N. Zufelato, E.P. Silveira-Lacerda, A. F. Bakuzis, Real-time infrared thermography detection of magnetic nanoparticle hyperthermia in a murine model under a non-uniform field configuration, Int. J. Hyperth.: Off. J. Eur. Soc. Hyperth. Oncol. North Am. Hyperth. Group 29 (2013) 752-767.

[13] M. Moreira, L.O. Murta, Jr., O. Baffa, Imaging ferromagnetic tracers with an ac biosusceptometer, Rev. Sci. Instrum. 71 (2000) 7. 